\title{
A Case of Boerhaave's Syndrome Presenting after a Trial of Non-Invasive Ventilation
}

\author{
M.S. Patel P.U. Chakraborty
}

Department of Intensive Care, St Helier Hospital, Surrey, UK of oesophageal rupture prior to commencing non-invasive ventilation, especially with regard to chest radiograph features.

Copyright $\odot 2009$ S. Karger AG, Basel

\begin{abstract}
Objective: To highlight the case of a patient with acute respiratory failure, whose diagnosis of Boerhaave's syndrome only became apparent after a trial of non-invasive ventilation. Clinical Presentation and Intervention: A 68-year-old female presented with a clinical picture of communityacquired pneumonia and exacerbation of asthma that was supported by radiological evidence of a large left-sided pleural effusion. Within $20 \mathrm{~h}$, she deteriorated and progressed to severe type 2 respiratory failure. After initiation of first non-invasive and then invasive ventilation, a tension pneumothorax developed. An emergency decompression of the chest revealed gastric contents in the left hemithorax. A diagnosis of Boerhaave's syndrome was made. Subsequent management included a thoracotomy, defunctioning oesophagectomy, and gastrostomy with ventilatory and inotropic support. However, despite best efforts, the severe systemic inflammatory response resulted in death 3 weeks after initial presentation. Conclusion: It is important to have an open diagnostic mind with a thorough review of investigations and therapy as a patient deteriorates. This case illustrates the importance of considering the remote possibility
\end{abstract}

\section{Introduction}

In 1724 Dr. Hermann Boerhaave described the first case of oesophageal perforation $[1,2]$, commonly known as Boerhaave's syndrome. Rapid diagnosis and therapy are essential in order to decrease mortality and morbidity $[3,4]$. Spontaneous oesophageal rupture occurs following a sudden increase in intraluminal pressure, commonly after retching or vomiting, usually after excessive food or alcohol intake $[2,5]$. The morbidity and mortality is most often due to the profound inflammatory response to gastric contents within the mediastinum, pleura and adjoining tissues [3]. The classic clinical presentation of Boerhaave's syndrome is the triad of severe retching or vomiting followed by acute and severe chest or epigastric pain with subcutaneous emphysema in the neck or chest after at least $1 \mathrm{~h}$ (the Mackler triad) [5-7]. Breath sounds are often reduced on the side of the perforation, which is most commonly left-sided [3]. When diagnosis is delayed, patients may become critically ill. Surgical intervention may become necessary, depending

\section{KARGER}

Fax +4161306 1234

E-Mail karger@karger.ch

www.karger.com
(C) 2009 S. Karger AG, Basel

1011-7571/09/0182-0155\$26.00/0

Accessible online at:

www.karger.com/mpp
Dr. M.S. Patel

Postgraduate Centre

St Helier Hospital, Wrythe Lane

Carshalton, Surrey, SM5 1AA (UK)

Tel. +44 208644 4343, E-Mail mehuliopatel@hotmail.com 
1

Fig. 1. Chest radiograph on initial presentation showing a left pleural effusion, left lower zone infiltrate, a small pneumomediastinum and subcutaneous air in the area of the neck and shoulders.

Fig. 2. Chest radiograph after developing respiratory failure and a trial of NIV. The air in the tissues has increased and a tension pneumothorax is now present on the left.
2

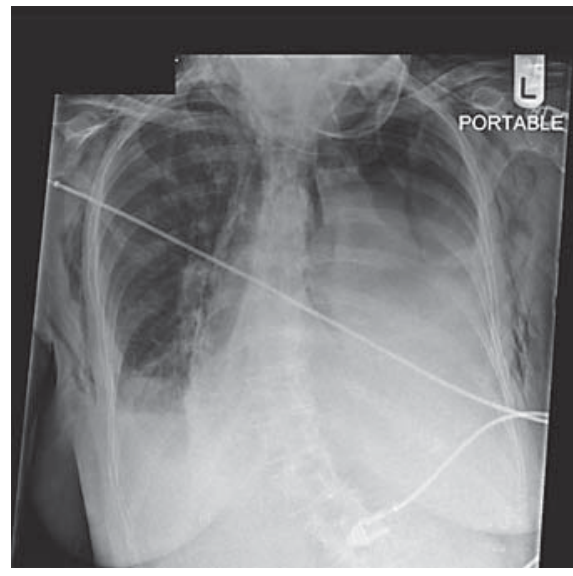

upon the extent and location of the injury. Today, spontaneous oesophageal perforation is rare, but still remains potentially fatal. Consequently, early diagnosis is essential in order to allow successful treatment. Because the symptoms are often non-specific, a high degree of vigilance is required to enable a rapid and accurate diagnosis. We report here a case of undetected Boerhaave's syndrome presenting to the Accident and Emergency Department of a district general hospital in the UK.

\section{Case Report}

A 69-year-old asthmatic woman presented to the Accident and Emergency Department of St Helier Hospital, Surrey, UK, with acute onset of vomiting, following the ingestion of an evening meal and sudden onset of sharp left-sided chest pain. She had recently been short of breath, resulting in increased use of her regular inhalers. She also complained of coryzal symptoms, with a cough productive of pale yellow sputum. Her appetite and eating habits had been unaffected, and her bowel motion earlier that day had been normal. Her chest pain was sufficient to require morphine analgesia in the Accident and Emergency Department.

Her past medical history included severe atopy with asthma that had recently been of worsening severity. However, her exercise tolerance was unhindered. She had severe eczema, which had been treated with steroids and immunosuppressants. The patient had attended a surgical outpatient clinic in the previous year; however, she declined intervention for an asymptomatic small epigastric hernia. Otherwise, there was no other gastrointestinal or surgical history. Her regular medications consisted of budesonide/formoterol and salbutamol inhalers. She had no relevant family history. The patient was normally independent, and had not seen her primary care physician for the preceding several months.

On initial presentation, she was systemically well and had no features of respiratory compromise. On auscultation, she had reduced air entry at the left base with some expiratory rhonchi and dullness to percussion at the left base. A low peak expiratory flow reading was recorded at $100 \mathrm{ml} / \mathrm{min}$. She was haemodynamically stable, and had no additional heart sounds or murmurs. Abdominal examination revealed tenderness in the left hypochondrium, extending to the lower anterior thoracic region; bowel sounds were present with no other signs of peritonitis. The only abnormality noted in the initial blood results was an increased white cell count of $27 \times 10^{9} / 1$, predominantly neutrophilic. An arterial blood gas showed that she was not hypoxaemic or acidotic. The electrocardiogram was unremarkable. On initial assessment, the chest radiograph revealed a moderately sized left pleural effusion with left lower lobe collapse; pneumothorax or pneumomediastinum was not identified (fig. 1).

Initial management was that of community-acquired pneumonia and exacerbation of her asthma. However, over the next $8 \mathrm{~h}$ she deteriorated, desaturating on $60 \%$ oxygen, with hypotension, tachycardia, tachypnoea and evidence of pulsus parodoxus. The Glasgow Coma Score had reduced from 15 to 14 due to confusion, and she had widespread wheezing with reduced breath sounds throughout both lung fields. She was rapidly transferred to the intensive care unit, where, following fluid resuscitation, she became haemodynamically stable. Her progressive type 2 respiratory failure and profound bronchoconstriction were treated with magnesium, aminophylline, hydrocortisone and a trial of noninvasive ventilation (NIV). The NIV was initiated with a fraction of inspired oxygen of 0.5 , a positive end-expiratory pressure of $5 \mathrm{~cm} \mathrm{H}_{2} \mathrm{O}$, and a pressure support level of $15 \mathrm{~cm} \mathrm{H}_{2} \mathrm{O}$. However, she rapidly became tired, exhibiting progressive central neurological impairment. A decision was made to progress to invasive ventilation. A chest radiograph immediately prior to this revealed a pneumomediastinum, large left-sided pneumothorax and subcutaneous emphysema (fig. 2). Whilst preparation for an intercostal chest drain was in progress, she developed a tension pneumothorax which required an emergency decompression, through which gastrointestinal contents were aspirated. More than $1.5 \mathrm{li}$ tres of similar fluid was collected on subsequent insertion of an intercostal chest drain. A diagnosis of oesophageal perforation, Boerhaave's syndrome, was made.

Following this diagnosis, and since the patient had presented less than $20 \mathrm{~h}$ previously, the decision was made to perform a thoracotomy. This revealed an $8-\mathrm{cm}$ rupture which was high in the oesophagus, with gastric contamination of the left hemithorax. A 
defunctioning oesophagectomy and gastrostomy were performed and drains inserted in the pleural cavity and mediastinum. However, the patient subsequently developed multiple organ failure requiring ongoing ventilatory, inotropic and renal support, finally succumbing 3 weeks later.

\section{Discussion}

This case highlighted some important clinical points. Although on admission the patient was managed appropriately for the clinical presentation, the possibility of oesophageal perforation was not initially considered. A confounding factor delaying diagnosis in this case was the profound bronchoconstriction. The original diagnosis of pneumonia in this patient, deteriorating with lifethreatening asthma, seemed to be consistent with the clinical features. It was only when she developed a pneumothorax and the presence of gastric aspirate from subsequent drainage that the diagnosis of oesophageal perforation was made.

Although some of the initial chest radiograph changes present were subtle and the most obvious feature was the left-sided pleural effusion, which was ascribed to a pneumonic process, further review showed that there was clearly subcutaneous emphysema and a very subtle but definite pneumomediastinum. Such signs are often overlooked in Boerhaave's syndrome when presenting early $[3-5,8]$. The patient gave a clear history of being rather well prior to the vomiting episode, other than some nonspecific upper airways symptoms. In the circumstances, therefore, pneumonia was high up on the list of differential diagnoses. However, retrospective closer review of the history and initial chest x-ray was consistent with a possible diagnosis of Boerhaave's syndrome on presentation. Had that been the case, nasogastric and intercostal chest drains would have been inserted on admission and definitive surgery contemplated prior to the decompensation. It highlights the importance of taking a thorough history and reviewing all the individual parts of an x-ray, even after an obvious explanatory pathology has been detected.

Another concern that this case raised is the use of noninvasive ventilation. It is a safe and effective tool that can be used to avoid the complications associated with endotracheal intubation in acute respiratory failure [9-11]. Although time consuming, NIV may reduce morbidity and mortality in selected groups. NIV preserves airway defence mechanisms, speech and swallowing, and affords flexibility in applying or removing ventilatory support.
The use of NIV, however, depends upon the type of acute respiratory failure (hypoxaemic or hypercapnic) and the underlying disease process. In this patient, it seemed an appropriate adjunct in the attempt to improve her respiratory function whilst trying to address the seemingly reversible component of her acute decline, the severe bronchoconstriction. Had this tactic been successful, it may have obviated the need for intubation and invasive mechanical ventilation that holds its own risks: nosocomial pneumonia, tracheal trauma, discomfort and the need for sedation. The rapid deterioration of the patient following NIV and the significant radiographic changes (namely, subcutaneous emphysema, pneumothorax and pneumomediastinum) thereafter suggests that the use of NIV may have in fact caused the cardiorespiratory decline in our patient. The raised intrathoracic and oesophageal pressures may have resulted in what was an initially small oesophageal perforation becoming more significant, and the progression of the pneumothorax under tension.

Retrospectively, it is most likely that our patient had a spontaneous oesophageal rupture which only became clinically apparent after the trial of NIV. Whilst tracheal intubation is a well-recognised cause of oesophageal perforation, our literature search revealed only 1 reported case of NIV causing oesophageal perforation de novo [12]. However, this case does support the proposition that NIV might have been implicated in the progression of the oesophageal perforation and the pneumothorax that had not been readily apparent previously.

\section{Conclusion}

This case illustrates the importance of considering the remote possibility of oesophageal rupture prior to commencing NIV, especially with regard to close inspection of the chest radiograph for findings which can be quite subtle. This is paramount in the management of patients presenting with an unusual constellation of clinical features that may be consistent with Boerhaave's syndrome.

Med Princ Pract 2009;18:155-158 


\section{References}

$>1$ Derbes VJ, Mitchell RE Jr: Hermann Boerhaave's Atrocis, nec descripti prius, morbi historia: the first translation of the classic case report of rupture of the esophagus, with annotations. Bull Med Libr Assoc 1955;43: 217-240.

-2 Bolooki H, Anderson I, Garcia Rivera C, Jude JR: Spontaneous rupture of the esophagus: Boerhaave's syndrome. Ann Surg 1971; 174:319-324.

$>3$ Curci J, Horman M: Boerhaave's syndrome: the importance of early diagnosis and treatment. Ann Surg 1976;183:401-408.

$\checkmark 4$ Bobo W, Billups W, Hardy J: Boerhaave's syndrome: a review of spontaneous rupture of the oesophagus secondary to vomiting. Ann Surg 1970;172:1034-1038.
5 Walker W, Cameron E, Walbaum P: Diagnosis and management of spontaneous transluminal rupture of the oesophagus (Boerhaave's syndrome). Br J Surg 1985;72: 204-207.

6 Pandey C, Bose N, Dash N, Singh N, Saxena $\mathrm{R}$ : Perioperative management of a patient presenting with a spontaneously ruptured esophagus. Can J Anaesth 2002;49:409412.

7 Kallis P, Belsham P, Pepper J: Spontaneous rupture of the oesophagus (Boerhaave's syndrome): conservative versus surgical management. J R Soc Med 1991;84:690-691.

8 Al-Mobeireek AF, Al-Kattan K: 'Coffee grounds' through the chest tube. Eur Respir J 2000;16:361-363.
9 Antonelli M, Pennisi M, Montini L: Clinical review: noninvasive ventilation in the clinical setting - experience from the last 10 years. Crit Care 2005;9:98-103.

10 British Thoracic Society Standards of Care Committee: Non-invasive ventilation in acute respiratory failure. Thorax 2002;57: 192-211.

11 Brochard L: Mechanical ventilation: invasive versus noninvasive. Eur Respir J Suppl 2003. 47:31s-37s.

12 Van de Louw A, Brocas E, Boiteau R, PerrinGachedoat D, Tenaillon A: Oesophageal perforation associated with noninvasive ventilation: a case report. Chest 2002;122: 1857-1858. 